\title{
Os pequenos perfumes fazem grandes frascos? Os efeitos do tamanho no desempenho municipal
}

\author{
Filipy Furtado Sell 1 \\ Leonardo Flach ${ }^{2}$ \\ Luísa Karam de Mattos ${ }^{3}$ \\ 1 Universidade Federal do Pará / Faculdade de Ciências Contábeis, Belém / PA - Brasil \\ 2 Universidade Federal de Santa Catarina / Programa de Pós-Graduação em Contabilidade, Florianópolis / SC - Brasil \\ 3 Universidade Federal de Santa Catarina / Programa de Pós-Graduação em Administração, Florianópolis / SC - Brasil
}

Esta pesquisa tem por objetivo evidenciar os efeitos direto e indireto do tamanho no desempenho dos municípios de Santa Catarina, em razão da discussão acadêmica quanto ao tamanho do município afetar o desempenho deste. Analisaram-se todos os municípios do estado de Santa Catarina, no período de 2005 a 2016, compreendendo três períodos eleitorais. A amostra é de 3.504 observações, com 293 municípios, no período de 12 anos. A análise de dados em painel foi estimada por mínimos quadrados generalizados factíveis (MQGF). Observa-se nos resultados indícios de que o tamanho afeta de forma direta e positiva o desempenho municipal e de forma indireta quando associado com a escolaridade do prefeito. Assim, nos municípios catarinenses, quanto maior for a população, maior será o IPTU arrecado per capita. E quanto maior for o tamanho da população associado a prefeitos com maior grau de escolaridade, maior será o desempenho municipal. Quando o desempenho é medido pelo percentual do IPTU arrecadado da receita fiscal total, não há efeito do tamanho e do tamanho associado à capacidade do prefeito no desempenho.

Palavras-chave: tamanho; desempenho; setor público; municípios.

\section{¿Los pequeños perfumes hacen grandes frascos? Los efectos del tamaño en el desempeño municipal}

Ante la discusión académica en cuanto a si el tamaño del municipio afecta el desempeño, esta investigación tiene por objetivo evidenciar el efecto directo e indirecto del tamaño en el desempeño de los municipios de Santa Catarina. Se analizaron todos los municipios del estado de Santa Catarina, en el período de 2005 a 2016, comprendiendo tres períodos electorales. La muestra es de 3.504 observaciones, con 293 municipios, en el período de 12 años. El análisis de datos en panel fue estimado por los mínimos cuadrados generalizados factibles (MCGF). En los resultados se observan indicios de que el tamaño afecta de forma directa y positiva el desempeño municipal y de forma indirecta cuando es asociado a la escolaridad del alcalde. Así, en los municipios catarinenses cuanto mayor sea la población mayor será el IPTU (impuesto sobre la propiedad y territorial urbano) recaudado per cápita y cuanto mayor el tamaño de la población asociado a alcaldes con mayor grado de escolaridad mayor será el desempeño municipal. Cuando el rendimiento se mide por el porcentaje del IPTU recaudado del ingreso fiscal total no hay efecto del tamaño y del tamaño asociado a la capacidad del alcalde en el desempeño.

Palabras clave: tamaño; rendimiento; sector público; municipios.

\section{Do good things come in small packages? The effects of size on municipal performance}

This research shows the direct and indirect effects of size on the performance of the Brazilian municipalities in the state of Santa Catarina, considering the academic debate around the issue. The research examined all municipalities of the state from 2005 to 2016, a period that comprised three municipal elections. The study sample consisted of 3,504 observations, with 293 municipalities, during these 12 years. The panel data analysis was estimated by Feasible Generalized Least Squares (FGLS). The results suggest that size affects municipal performance positively and directly. Also, size influences indirectly when considering the mayor's schooling, i.e., the larger the municipality and the higher the mayor's schooling, the better the municipal performance. The analysis of performance based on municipal tax (IPTU) collected per capita showed that, the larger the population, the higher the IPTU per capita, which means that larger municipalities tend to show better performance. Finally, the analysis of performance based on the percentage of IPTU levied from total tax revenue showed that size and size associated with the mayor's capability did not affect performance.

Keywords: size; performance; public sector; municipalities. 


\section{INTRODUÇÃO}

A New Public Management (NPM) reforma a gestão pública de perspectivas burocráticas para gerencialistas, estimulada pela globalização e pelas privatizações. A filosofia é de um Estado facilitador das prestações de serviços públicos, ao invés de prestador direto. Há também a defesa da absorção gradual de conceitos da contabilidade privada para a abordagem pública, como: eficiência, eficácia, foco na tomada de decisão e inovações no sistema contábil (Lapsley, 1999; Parker \& Gould, 1999; Van der Hoek, 2005).

A reforma do setor público parte de uma demanda internacional que, além de observar as características de eficiência e foco na tomada de decisão (Van der Hoek, 2005), visa a comparação entre países, com o objetivo de melhorar a gestão e o desempenho, fortalecer o controle das contas públicas e facilitar a responsabilização dos gestores pela má gestão dos recursos públicos (Campbell, 1993; Peters \& Savoie, 1996). Adicionalmente, Romzek (2000) afirma que apesar de se posicionar a favor da eficiência no setor público, o foco na reforma está nos desafios e nos impactos na prestação de contas dos gestores públicos.

Nesta linha, autores destacam que o tamanho da organização pode influenciar seu desempenho e que pequenas unidades governamentais tendem a ser mais eficientes, visto a proximidade com a população facilitar a compreensão de suas necessidades (Flynn, 1993; Grosskopf \& Yaisawarng, 1990; Holzer et al., 2009). Assim, Schumacher (1973) criou o termo small is beautifulI, caracterizando a fragmentação de municípios por países em desenvolvimento.

Na literatura científica, alguns estudos já encontraram relação de causalidade sobre o efeito do tamanho no desempenho no setor público. Entre os estudos que encontraram relação positiva de causalidade, constam: Avellaneda e Gomes (2015, 2017); Christenson e Sachs (1980); Smith e Meier (1994); Whetten (1978). Mas este ainda não é um consenso, pois os seguintes autores encontraram relação negativa de causalidade: Fowler e Walberg (1991); Heck e Mayor (1993); Sharkansky (1967). Além disso, Dean e Peroff (1977) encontraram relação neutra. Portanto, existe esta lacuna na literatura científica.

Desse modo, emerge a seguinte questão de pesquisa: qual é o efeito direto e indireto do tamanho no desempenho municipal? Assim, esta pesquisa tem por objetivo evidenciar o efeito direto e indireto do tamanho no desempenho dos municípios de Santa Catarina, nos anos de 2005 a 2016.

O estudo ampliará a pesquisa de Avellaneda e Gomes (2015), por aplicar o método adotado nos municípios do estado de Santa Catarina, nos anos de 2005 a 2016, compreendendo três períodos eleitorais. A relevância do estado de Santa Catarina se deve ao fato de manter-se estável perante os demais estados brasileiros, no período contemporâneo de instabilidade política e econômica.

Uma importante hipótese desta pesquisa consiste em verificar a relação entre escolaridade de prefeitos e maior arrecadação do Imposto Predial e Territorial Urbano (IPTU). O intuito é realizar uma argumentação sobre a relação lógica entre as variáveis, para compreender se há causalidade ou se a escolaridade pode ser efeito de outras variáveis, como urbanização, ideologia partidária ou profissionalização da administração direta municipal. Marenco, Strohschoen e Joner (2017) encontraram relação forte e estatisticamente significativa entre a proporção de estatutários com formação superior na administração direta e a arrecadação de IPTU, mesmo em pequenos e micro municípios. Além disso, Lowi (2009) explica que o imposto patrimonial como IPTU consiste em política redistributiva, que implica jogo de soma-zero, com perdedores e conflito. 
Como justificativa teórica, ressalta-se a contribuição para a literatura com a análise longitudinal, pois estudos longitudinais conseguem superar efeitos específicos das organizações, mitigando resultados tendenciosos pelo efeito do tempo (Adhikari, Derashid \& Zhang, 2006). Além de ser considerado fundamental para dedução de inferências causais (Hedeker \& Gibbons, 2006), os estudos longitudinais mitigam problemas de análises estatísticas, pois ofertam maior variabilidade de dados e possuem como resultado maior suavidade nos dados a serem analisados, em comparação com as análises individuais (Fávero \& Belfiore, 2017).

Além da contribuição para a literatura, do ponto de vista da justificativa prática, o estudo contribui para a sociedade, por evidenciar o efeito do tamanho e de variáveis de controle (material humano, receita própria municipal, características gerenciais dos gestores e características políticas) no desempenho, vistos como relevantes para análise, diante do cenário contemporâneo brasileiro de incerteza econômica e política.

\section{REVISÃO TEÓRICA: 0 EFEITO DO TAMANHO NO DESEMPENHO MUNICIPAL}

O conceito de desempenho no setor público foi desenvolvido na literatura científica com base em fatores sociodemográficos, políticos, econômicos. Dentre as influências políticas no desempenho, os estudos se referem a apoio partidário (Doig \& Hargrove, 1990), mecanismos de controle (Clingermayer \& Wood, 1995), controle social e participação dos cidadãos (Blair, 2000), ideologia do governo (Swank, 2002), motivação dos políticos (Anderson, 2003; Gibson \& Lehoucq, 2003) e competitividade eleitoral (Holbrook \& Van Dunk, 1993). Entre os determinantes econômicos para o desempenho no setor público, os estudos identificaram a influência de orçamentos, Produto interno bruto (PIB) e nível de desenvolvimento. E como explicação sociodemográfica para o desempenho no setor público, as pesquisas científicas apontam para natureza da população-alvo e para o tamanho da organização (Avellaneda, 2009; Durant \& Legge, 1993; Walker, Damanpour \& Avellaneda, 2009). Embora este conjunto de influências políticas, econômicas e sociodemográficas tenha um enfoque mais voltado aos fatores externos à organização (nível macro), há também autores que apontam para o nível micro. Neste caso, o desempenho é influenciado também por fatores internos, inerentes aos potenciais do indivíduo, e também do grupo, que exerce influência e controle por parte de um gestor público eleito. De fato, nenhum grupo de atores políticos torna-se mais importante para a operação e para o desempenho nos órgãos públicos do que os funcionários eleitos (Pandey \& Moynihan,2006).

Gooding e Wagner (1985) já demonstravam o interesse de pesquisadores em analisar o efeito do tamanho da organização no desempenho obtido. Os autores mostram que pesquisadores organizacionais se preocupam com a estrutura da organização, sendo o tamanho uma das características mais difundidas nas relações organizacionais. Estudos da área de economia costumam relacionar o tamanho das organizações com economias de escala, tendo relações significativas positivas e negativas e variações quanto à especificidade do tamanho (organizações maiores e menores). Estudos na área de psicologia social relacionam o tamanho do grupo com o desempenho do grupo. Os achados desses estudos ainda demonstram resultados variados quanto à relação proposta, pois encontram geralmente relações insignificantes ou negativas, ou ainda elevados custos de coordenação do grupo.

Observa-se que, no âmbito de pesquisas que utilizam como base de estudo os municípios, há uma tendência para resultados que demonstram que quanto maior o tamanho dos municípios, maiores serão as responsabilidades com os serviços sociais a serem ofertados (Stein, 1990). Deste modo, Stein (1990) explica que, com a expansão da cobertura dos municípios, haveria um aumento nos custos 
dos serviços prestados, devido ao aumento na força de trabalho demandada para disponibilizar os serviços à população.

Neste sentido, Meier e O'Toole (2002) apresentam que quanto maior for o tamanho do distrito, maior será a demanda de serviços. E, com isso, maior será a força de trabalho necessária para a execução dos serviços públicos. Isto demandaria maior quantidade de recursos. Uma alternativa para melhorar o desempenho e a organização dos municípios para enfrentar essa nova demanda de serviços, causada pela sua expansão, consiste na fusão de municípios (Steiner, 2003).

A fusão, além de aumentar o tamanho territorial do município, aumenta o potencial de obtenção de recursos e a disseminação de novas formas de governar, visto que em municípios maiores os conceitos e princípios da New Public Management (NPM) são mais generalizados (Steiner, 2003). Ressalta-se que a generalização da NPM dissemina a eficiência na utilização dos recursos públicos e responsabiliza os gestores pela má gestão do erário (Lapsley, 1999; Parker \& Gould, 1999; Romzek, 2000, Van der Hoek, 2005).

Observando o cenário de aumento do tamanho dos municípios, Damanpour, Walker e Avellaneda (2009) destacam que o tamanho afeta a relação das características organizacionais e da demanda de recursos no desempenho. Os achados de Damanpour et al. (2009) indicam que quanto maior for o tamanho organizacional e a urbanização do município, maior será o desempenho. Mas quanto maior for a necessidade e a diversidade de serviços a serem prestados, menor será o desempenho.

Um contraponto para que as organizações municipais maiores tenham maior desempenho é o argumento exposto por Holzer et al. (2009). Os autores propõem que na relação entre tamanho e desempenho, quanto menores forem os municípios, maior será a proximidade dos gestores com as necessidades da população, tendendo a obter maior desempenho. Todavia, nessa reflexão cabe destacar que, ao analisar o desempenho per capita em relação ao tamanho da população, a tendência é: quanto maior e menor o tamanho do município menor será o desempenho. Assim, os picos de tamanho municipal (maior e menor) relacionam-se negativamente com o aumento no desempenho. Os municípios de tamanho médio tendem a apresentar melhor desempenho (Drew, Kortt \& Dollery, 2014; Holzer et al., 2009).

Neste sentido, Holzer et al. (2009) concluíram que municípios com população entre 25 mil e 250 mil habitantes são mais eficientes que municípios com mais de 250 mil habitantes. Apesar dessa pesquisa demonstrar uma tendência de municípios com menor número de habitantes serem mais eficientes que municípios com mais habitantes, Avellaneda e Gomes (2015) destacam que não há um consenso na literatura e julgam que sejam necessários mais estudos na área.

O indicador de desempenho no estudo de Avellaneda e Gomes (2015) foi a arrecadação de impostos sobre a propriedade. Com base na inconsistência da literatura entre a influência do tamanho dos municípios no desempenho, e a tendência de municípios menores serem mais eficientes (Holzer et al., 2009), tem-se a primeira hipótese do estudo:

$H_{1}$ - O tamanho dos municípios é positivamente relacionado com o desempenho (arrecadação de impostos próprios).

Além de observar a relação direta entre o tamanho do município com o desempenho, verificar-se-á a relação indireta, visto que municípios de médio porte podem possuir densidade econômica, maior quantidade de material humano e valores aplicados no recolhimento de recursos próprios (Avellaneda \& Gomes, 2015; Drew et al., 2014). 
Deste modo, será observada a relação inversa entre o tamanho e o desempenho, com base no U invertido, considerando as características dos municípios médios de não terem a mesma burocracia que os municípios grandes enfrentam, e tampouco a proximidade dos municípios pequenos (Avellaneda \& Gomes, 2015). Assim:

\section{$H_{2}$ - O tamanho populacional é curvilineamente associado ao desempenho municipal.}

No contexto do desempenho municipal, observa-se que as características do líder de uma organização afetam os recursos materiais e humanos, por meio de suas decisões e definições de estratégias (Avellaneda, 2012). Lynn (1996) ressalta que o líder obtém todo o seu conhecimento por meio de sua experiência e pela educação formal.

Evidências na literatura gerencial descrevem que há um efeito positivo entre o capital humano no retorno de vendas. Este efeito é maior em empresas grandes do que em empresas pequenas (RocaPuig, Beltrán-Martín \& Segarra Cipres, 2011). Traçando um paralelo entre empresas e municípios, percebe-se que governar um município maior exige maior coordenação, comportamento estratégico, diferenciação organizacional e priorizações no orçamento (Avellaneda \& Gomes, 2015; Meier \& O'Toole, 2002; Steiner, 2003).

Deste contexto, destaca-se a terceira hipótese desta pesquisa:

$H_{3}$ - O impacto do capital humano de um gestor sobre o desempenho municipal é moderado positivamente pelo tamanho da população.

Assim, tendo o exposto neste tópico, reforça-se o desenvolvimento desta pesquisa contribuindo para a discussão do efeito do tamanho no desempenho. Estes resultados poderão ser utilizados como base para a tomada de decisão em planejamentos de expansões e desenvolvimentos municipais.

\section{MÉTODO}

Visando atingir o objetivo de evidenciar o efeito direto e indireto do tamanho no desempenho municipal, posiciona-se esta pesquisa no paradigma funcionalista (Burrell \& Morgan, 1979), observando a tipologia de pesquisa descritiva, com abordagem quantitativa dos dados (Sampieri, Collado \& Lucio, 2013).

Os dados foram coletados nos sites da Secretaria do Tesouro Nacional (STN), do Instituto Brasileiro de Geografia e Estatística (IBGE), do Tribunal Superior Eleitoral (TSE), do Tribunal de Contas do Estado de Santa Catarina (TCE-SC) e nos portais de transparência dos municípios analisados. A população da pesquisa compreende todos os municípios do estado de Santa Catarina, contemplando 295 municípios, de 2005 a 2016.

Convém registrar que devido à falta de dados cobrindo todo o período analisado, os municípios de Balneário Rincão e Pescaria Brava (sem dados de 2004 a 2011) foram excluídos da amostra. Logo, a amostra final reuniu 293 municípios, correspondendo a 3.504 observações. Ressalta-se que os municípios excluídos foram criados em 2003, sendo efetivamente instalados em 2013.

Para facilitar a compreensão, evidencia-se na Tabela 1 as variáveis dependentes, independentes e de controle, conforme observado por Avellaneda e Gomes (2015). 


\section{TABELA 1 VARIÁVEIS PESQUISADAS}

\begin{tabular}{|c|c|c|}
\hline \multicolumn{3}{|c|}{ Variável Dependente } \\
\hline Variável & Medida & Autor \\
\hline 1 - IPTU per capita & IPTUpc $=\frac{\text { IPTU Arrecadado }}{\text { População }}$ & $\begin{array}{l}\text { Avellaneda e Gomes } \\
\qquad \text { (2015) }\end{array}$ \\
\hline $\begin{array}{l}2 \text { - Participação do IPTU na receita } \\
\text { fiscal total }\end{array}$ & IPTUrt $=\frac{\text { IPTU pc }}{\text { Receita Fiscal Total }}$ & \\
\hline \multicolumn{3}{|c|}{ Variável Independente } \\
\hline $\begin{array}{l}3 \text { - Tamanho do município } \\
4 \text { - Capacidade do prefeito referente } \\
\text { à idade (IP) }\end{array}$ & $\begin{array}{c}L P=\text { Log População } \\
I P=\text { Log Idade do Prefeito }\end{array}$ & $\begin{array}{l}\text { Avellaneda e Gomes } \\
\qquad(2015)\end{array}$ \\
\hline $\begin{array}{l}5 \text { - Capacidade do prefeito referente } \\
\text { à escolaridade (EP) }\end{array}$ & $\begin{array}{l}\text { Nível de escolaridade do prefeito, sendo obtido pela escala: } \\
\begin{array}{c}1 \text { - Mal consegue ler seu próprio nome; } \\
2 \text { - Ensino Fundamental incompleto; } \\
3 \text { - Ensino Fundamental; } \\
4 \text { - Ensino Médio incompleto; } \\
5 \text { - Ensino Médio completo; } \\
6 \text { - Graduação incompleta; } \\
7 \text { - Graduação completa; } \\
8 \text { - Pós-graduação. }\end{array}\end{array}$ & \\
\hline \multicolumn{3}{|c|}{ Variável de Controle } \\
\hline $\begin{array}{l}6 \text { - Alinhamento partidário entre } 0 \\
\text { governo e o prefeito (AP) }\end{array}$ & $\begin{array}{l}\text { Governador e prefeito são do mesmo partido político? } \\
\qquad 1 \text { - Sim / } 2 \text { - Não }\end{array}$ & $\begin{array}{l}\text { Avellaneda e Gomes } \\
\qquad(2015)\end{array}$ \\
\hline 7 - Ex-prefeito (ExP) & $\begin{array}{c}0 \text { prefeito possui eleição anterior? } \\
1 \text { - Sim / } 2 \text { - Não }\end{array}$ & \\
\hline 8 - Direção partidária (DP) & $\begin{array}{l}\text { Qual a direção partidária do prefeito? } \\
1 \text { - Esquerda / } 2 \text { - Direita / } 3 \text { - Centro }\end{array}$ & \\
\hline 9 - PIB per capita 0 & $P I B p c=\frac{P I B}{\text { População }}$ & \\
\hline 10 - Densidade populacional (DP) & $D P=\frac{\text { População }}{\text { Área do município }\left(\mathrm{km}^{2}\right)}$ & \\
\hline 11 - Proximidade com a capital & Distância em km do município em relação a capital. & \\
\hline $\begin{array}{l}12 \text { - Medida de desempenho de } \\
\text { cobrança }\end{array}$ & IPTU Arrecadado $_{t-1}$ & \\
\hline
\end{tabular}

Fonte: Dados da pesquisa. 
Destaca-se na Tabela 1 a variável de controle Direção Partidária por utilizar a metodologia de Carreirão (2006) para posicionar os partidos políticos brasileiros. Deste modo, classificam-se como partidos de direita: DEM, PR, PP, PFL, PRN, PDC, PL, PTB, PSC, PSP, PRP, PSL, PSD e PRONA, bem como o PMDB e PSDB (mesmo tendo características centrais, pela orientação conservadora considerou-se como partido de direita); com partidos de esquerda: PT, PDT, PPS, PC do B, PSB, PV, PSTU, PCO e PMN; e como partido de centro: PHS, PPS, PRB, PRTB, PSD, PSDC, PT DO B, PTC e PTN.

Quanto ao modelo estatístico a ser utilizados para análise, tem-se que, dada a natureza transversal do conjunto de dados, da variável dependente (modelo 1), os modelos a seguir foram estimados pelos mínimos quadrados ordinários (MQO). O modelo da variável dependente (modelo 2) inclui as mesmas variáveis explicativas, como segue:

$$
\begin{aligned}
& I P T U p c=\alpha+\beta_{1}(L P)+\beta_{2}(L P)^{2}+\beta_{3}(I P)+\beta_{4}(E P)+\beta_{5}(L P \star I P)+\beta_{6}(L P \star E P)+\beta_{7}\left(X_{i}\right)+\beta_{8} e \\
& \text { IPTUrt }_{i}=\alpha+\beta_{1}(L P)+\beta_{2}(L P)^{2}+\beta_{3}(I P)+\beta_{4}(E P)+\beta_{5}(L P \star I P)+\beta_{6}(L P \star E P)+\beta_{7}\left(X_{i}\right)+\beta_{8} e
\end{aligned}
$$

em que, IPTUrt ${ }_{i}$ é o IPTU per capita; “ $X$ ” refere-se aos determinantes socioeconômicos conhecidos e esperados das receitas municipais (variáveis de controle), "EP" é o nível de escolaridade do prefeito; "IP" é o logaritmo da capacidade do prefeito referente à idade; " $L P$ " é o logaritmo do tamanho da população; " $e$ " é o termo de erro.

Ocorre que no modelo 1, ao elaborar os testes para verificar a presença de heterocedasticidade, utilizando o teste de Poi e Wiggins (2001) e o teste de Wald, já que o melhor estimado é Efeitos Fixos (pelos testes $F$ de Chow e Hausman); e a autocorrelação com o teste de Wooldrige (Fávero \& Belfiore, 2017), obteve-se nos resultados dos testes que há presença de heterocedasticidade e autocorrelação nos dados.

Gujarati e Porter (2011) já orientavam que é factível e corriqueiro que se tenha problemas de heterocedasticidade e autocorrelação em dados em painel. Assim, uma alternativa para corrigi-las é reestimar o modelo, utilizando os estimadores dos mínimos quadrados generalizados factíveis (MQGF) (Fávero \& Belfiore, 2017; Gujarati \& Porter, 2011).

Deste modo, o modelo 1 foi reestimado utilizando os estimadores MQGF com autocorrelação sendo controlados pelo método de Durbin-Watson. Já o modelo 2, com base nos testes $F$ de Chow e no de Lagrange de Breush-Pagan, deve-se utilizar método Pools ou dados empilhados (Fávero \& Belfiore, 2017). Em face aos mesmos problemas de heterocedasticidade e autocorrelação, o método Pools foi estimado com base nos mínimos quadrados generealizados factíveis (MQGF).

Os modelos foram executados no programa Stata ${ }^{\circledR}$, sendo os resultado apresentados no próximo tópico. 


\section{ANÁLISE DOS DADOS E DISCUSSÃO DOS RESULTADOS}

Com base nas variáveis apresentadas na Tabela 1, ressalta-se a análise descritiva de algumas variáveis, quais sejam: com média de $\mathrm{R}$ \$209,04, mínimo de $\mathrm{R}$ \$,09 (Brusque) e máximo de $\mathrm{R} \$ 560.059,03$ (Biguaçu), observa-se alta variação nos municípios catarinenses, corroborando com os municípios de Minas Gerais, face a pesquisa de Avellaneda e Gomes (2015). A variável IP, com média de 49,40 anos, mínimo de 24 (Mafra) e máxima de 80 anos (Barra Velha), também se assemelha aos municípios de Minas Gerais por possuírem média de 48, mínimo 25 e máximo 85 anos (Avellaneda \& Gomes, 2015).

A variável EP com média de 5,44, demonstra que na média os prefeitos possuem o ensino médio completo e, com base nos valores mínimos e máximos tem-se que, cinco municípios possuem prefeitos que mal conseguem ler seu próprio nome (Bombinhas, Luiz Alvez, Rio das Antas, Romelândia e Timbó Grande) e um município possui prefeito com pós-graduação.

Visando aprimorar a informação, e por ser o único município com prefeito com pós-graduação, foi necessária a confirmação dos dados por meio da assessoria do prefeito de Biguaçu e foi constatado que ele possui especialização lato sensu em Gestão em Segurança Pública. Ressalta-se que nenhum prefeito possui especialização stricto sensu.

Os dados da escolaridade avançam na pesquisa de Avellaneda e Gomes (2015), tendo em vista que os pesquisadores posicionaram o máximo da métrica em sete pontos, tendo como máximo a graduação completa. Comparando as médias da escolaridade dos prefeitos dos municípios de Santa Catarina e de Minas Gerais, nota-se similitude nas amostras visto que na média os prefeitos dos dois estados possuem ensino médio completo.,

A estatística descritiva da variável alinhamento partidário entre o prefeito e o governador do estado apresenta valor médio de 1,77, assim observa-se uma tendência dos prefeitos não serem do mesmo partido do governador. Nota-se, também, como tendência ideológico-partidária dos prefeitos de Santa Catarina serem de direita (média de 1,97).

Os resultados das variáveis alinhamento partidário e ideologia partidária corroboram com o estudo Avellaneda e Gomes (2015), pois em Minas Gerais foi encontrada a mesma tendência que a de Santa Catarina. Neste ponto, destaca-se uma limitação das pesquisas (a presente pesquisa e a de Avellaneda e Gomes) visto que não foram analisadas as coligações partidárias, ou seja, mesmo que o prefeito não esteja no mesmo partido do governador pode haver alinhamento partidário pelas coligações.

Ressalta-se que não há um consenso nas coligações para eleições municipais e estaduais, ou seja, partidos coligados para eleições municipais podem não repetir a coligação para as eleições estaduais. Findando as análises descritivas das variáveis, observa-se que no estado de Santa Catarina tende-se à reeleição dos prefeitos (média 1,70), divergindo do estado de Minas Gerais que não há essa tendência (média 0,37).

Cabe destacar que os dados apresentados por Avellaneda e Gomes (2015) referem-se aos anos de 2005 a 2007, abarcando 787 observações no estado de Minas Geais, enquanto a pesquisa atual refere-se ao intervalo entre 2005 e 2016, contemplando 3.504 observações no estado de Santa Catarina, logo devem-se resguardar as peculiaridades socioeconômicas elapsos temporais não controlados para fins de comparação.

Prosseguindo na análise estatística dos dados, observa-se primeiro a Tabela 2 na qual são feitas as correlações entre as variáveis apresentadas. Nota-se que, exceto as relações AP e D.Part (-0,4240); LP e Dens.Pop $(0,5462)$ e Desemp.IPTU e AP $(0,6426)$, significantes a $1 \%$, possuem correlação moderada, as correlações apresentam-se de bem fraca a fraca (Fávero \& Belfiore, 2017).

Importa baixa correlação, pois as variáveis com alta correlação podem omitir/potencializar os resultados ou enviesar as análises (Fávero \& Belfiore, 2017). 
RAP | Os pequenos perfumes fazem grandes frascos? Os efeitos do tamanho no desempenho municipal

$\simeq$

8

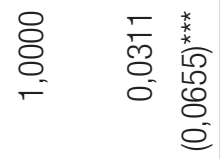

으

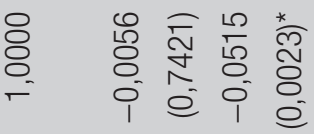

๑

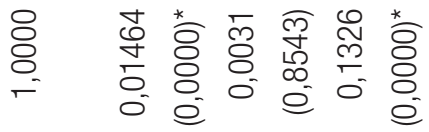

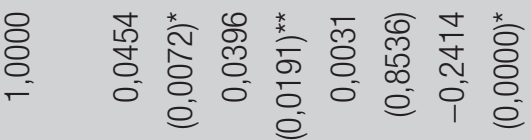

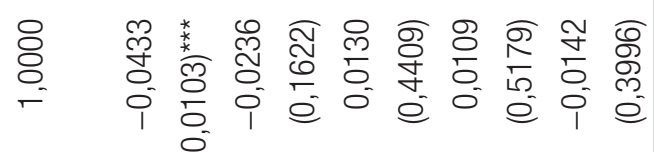

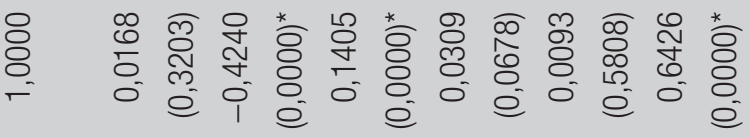

ก

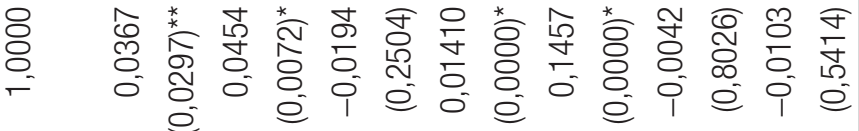

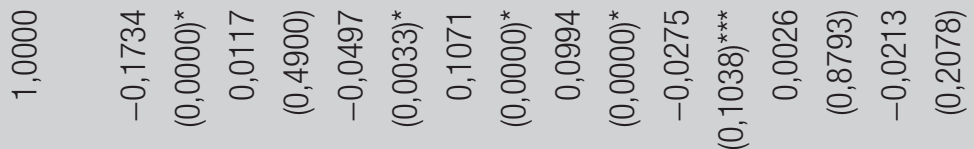

m

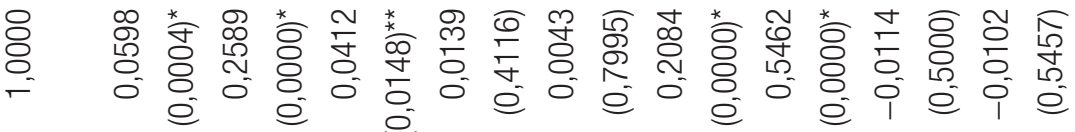

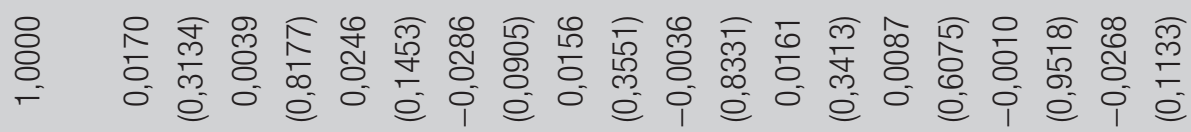

$\sim$

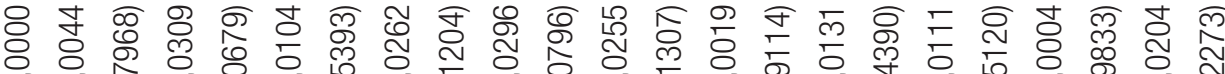

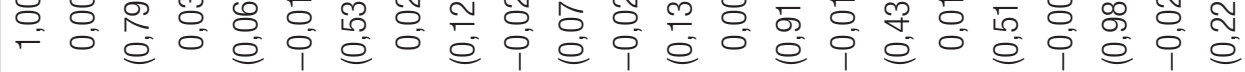

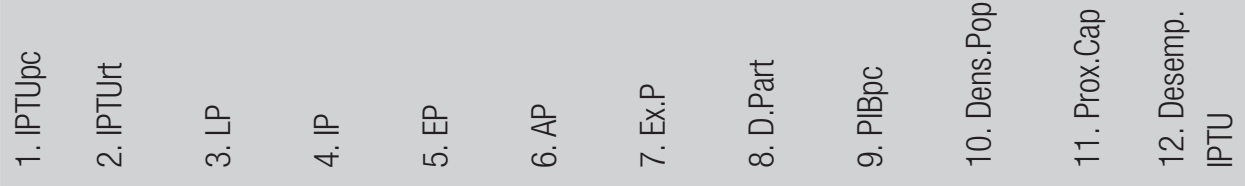


A correlação entre o LP e a Dens.Pop era esperada, pois quanto maior a população, maior será a densidade populacional. O resultado negativo entre AP e D.Part chama a atenção por sugerir que quando há alinhamento partidário entre o prefeito e governador ocorre direcionamento partidário inverso. Esta relação, aparentemente sem lógica, pode ser explicada por coligações partidárias em eleições municipais, contemplando em uma mesma bandeira de campanha política partidos de esquerda, direita e de centro.

Já a Desemp.IPTU, medida pelo logaritmo natural da arrecadação do IPTU no ano anterior, está positivamente associada ao alinhamento partidário entre o prefeito e o governador. Nesta relação específica, não se observa na literatura explicação factível e ressalta-se que é uma relação entre duas variáveis de controle que fazem sentido apenas em relação às variáveis dependentes.

As próximas análises prendem-se às Tabelas 3 e 4, nas quais apresentam-se os resultados dos modelos 1 e 2. Ressalta-se que, para melhor ajuste dos modelos de análises propostos, foram excluídas as variáveis densidade populacional (Dens.Pop), proximidade com a capital do estado (Prox.Cap) e desempenho do IPTU arrecadado no ano anterior (Desemp.IPTU). Assim, o elemento "' da equação dos dois modelos será composto pelas variáveis de controle alinhamento partidário (AP), Ex-Prefeito (Ex.P) e PIB per capta ().

Ressalta-se que os modelos 1 e 2 foram estimados pelos mínimos quadrados generealizados factíveis (MQGF); e foram calculados de três formas distintas: a primeira comtempla estritamente as variáveis dependentes, independentes e controle sem as interações e sem termos quadráticos; na segunda adicionou-se a variável com interação quadrática; e na terceira adicionaram-se as variáveis interações entre si.

\section{TABELA 3 MODELO 1}

\begin{tabular}{|c|c|c|c|}
\hline IPTUpc & MQGF & $\begin{array}{l}\text { Modelo com termos } \\
\text { quadráticos }\end{array}$ & $\begin{array}{c}\text { Modelo com interação de } \\
\text { termos }\end{array}$ \\
\hline \multicolumn{4}{|c|}{$H_{1}$ - Tamanho do município } \\
\hline LP & $257,8015(0,081)^{\star \star \star}$ & $-270,7618(0,876)$ & $1423,5480(0,670)$ \\
\hline \multicolumn{4}{|c|}{$\mathrm{H}_{2}$ - Efeito curvilíneo do tamanho } \\
\hline $\mathrm{LP}^{2}$ & 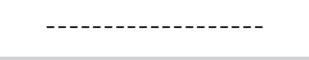 & $27,4870(0,760)$ & $-40,4380(0,673)$ \\
\hline \multicolumn{4}{|l|}{ Capacidade do prefeito } \\
\hline IP & $-413,4802(0,659)$ & $-393,1385(0,676)$ & $3260,3430(0,645)$ \\
\hline $\mathrm{EP}$ & $117,4420(0,225)$ & $119.2618(0,219)$ & $-1669,947(0,050)^{\star \star}$ \\
\hline \multicolumn{4}{|c|}{$\mathrm{H}_{3}$ - Efeitos moderadores } \\
\hline$L P^{*} \mid \mathrm{P}$ & 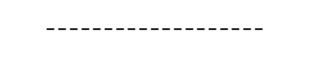 & 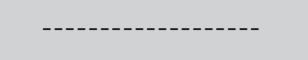 & $-403,0164(0,595)$ \\
\hline$L P * E P$ & 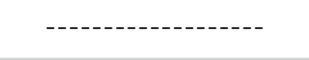 & 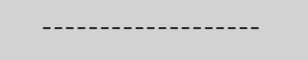 & $201,1074(0,034)^{\star \star}$ \\
\hline \multicolumn{4}{|l|}{ Variáveis de controle } \\
\hline AP & $-637,3110(0,094)^{\star \star \star}$ & $-363,6453(0,094)^{\star \star \star}$ & $-628,2533(0,098)^{\star \star \star}$ \\
\hline
\end{tabular}




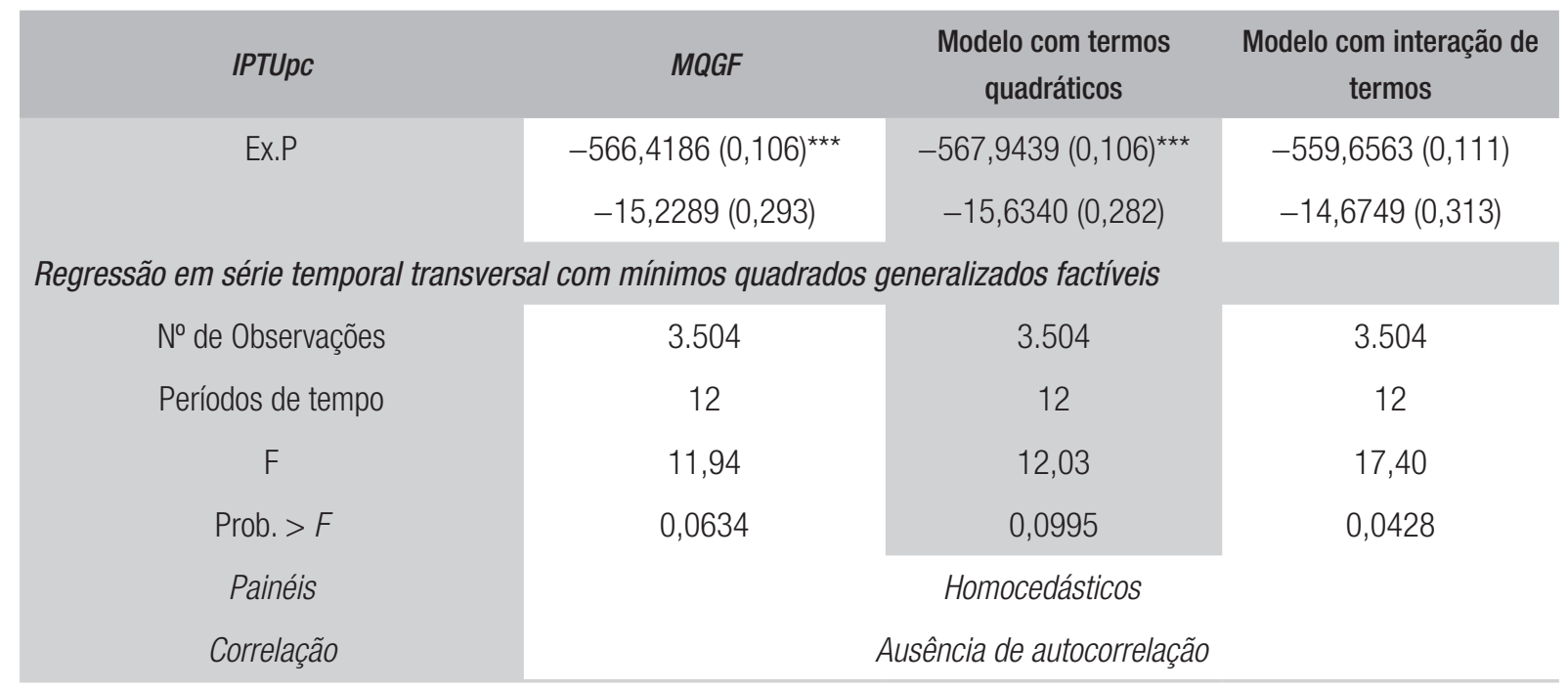

Nota: ${ }^{* *} p<0,10 ;{ }^{* *} p<0,05 ;{ }^{*} p<0,01$.

Fonte: Dados da pesquisa.

Observa-se que na primeira forma de análise do modelo 1, tem-se a variável LP influenciando positivamente a variável dependente com $10 \%$ de confiança. Este resultado demonstra que quanto maior a população do município, maior será o seu desempenho, ou maior será o volume de arrecadação de IPTU per capita do município.

Com esse resultado, confirma-se a hipótese de que o tamanho do município é positivamente relacionado com o desempenho na arrecadação de impostos próprios, no caso desta pesquisa, o IPTU. Este resultado corrobora com os estudos de Avellaneda e Gomes (2015), Christensen e Sachs, (1980), Smith e Meier (1994) e Whetten (1978) e se diverge da pesquisa de Holzer et al. (2009), pois estes defendem a perspectiva de que municípios menores são mais eficientes na arrecadação por não precisarem de uma estrutura extensa de controle e porque a administração está mais próxima da população, conhecendo suas demandas e anseios.

Ressalta-se que com as interações quadráticas e a interação entre termos, não há significância estatística na relação entre o tamanho da população e o desempenho. Neste giro, observa-se que em nenhuma das formas de análise do modelo 1 e 2, a hipótese $\mathrm{H}_{2}$ possui sustentação estatística para ser confirmada. Deste modo, rejeita-se a hipótese de que o tamanho populacional é curvilineamente associado ao desempenho municipal.

Mesmo que se tenha base teórica que suporte a hipótese de que municípios de tamanho médio possuem maior desempenho, por não demandarem uma estrutura burocrática extensa como nos municípios grandes e nem haver a proximidade com a população como nos municípios pequenos, conhecendo suas necessidades (Drew et al., 2014), a rejeição da $\mathrm{H}_{2}$ corrobora com a pesquisa de Avellaneda e Gomes (2015) porque também não possui significância estatística na relação proposta. Assim, nos estados de Santa Catarina e de Minas Gerais reforça-se que quanto maior o município, maior será o desempenho.

Ao testar se o impacto do capital humano de um gestor sobre desempenho municipal é moderado positivamente pelo tamanho da população, os resultados demonstram que quanto maior a escolaridade do prefeito (EP), associada a um maior tamanho populacional (LP), maior será o desempenho municipal na arrecadação de IPTU per capita. 
Este achado corrobora com Avellaneda e Gomes (2015), Meier e O'Toole (2002), Steiner (2003), pois quanto maior a capacidade humana do gestor relacionada à escolaridade, maior será sua capacidade de empreender no município para captação de organizações, e consequentemente novos empreendedores, novas oportunidades, contribuindo para o desenvolvimento municipal. Neste sentido, Lynn (1996) destaca que uma das formas de o líder obter conhecimento para gestão é por meio da educação formal.

Avellaneda (2012) afirma que as características do gestor organizacional afetam os recursos materiais e humanos por intermédio das suas decisões. Logo, quanto maior o município, maior será a exigência de coordenação e comportamento estratégico do prefeito para aumentar o desempenho na arrecadação (Avellaneda \& Gomes, 2015; Meier \& O'Toole, 2002; Steiner, 2003).

Apresentados e discutidos os resultados do modelo 1, na sequência, apresenta-se a Tabela 4 para demonstrar o resultado das três formas de análise do modelo 2 .

\section{TABELA 4 MODELO 2}

\begin{tabular}{|c|c|c|c|}
\hline IPTUrt & POOLS & $\begin{array}{l}\text { Modelo com termos } \\
\text { quadráticos }\end{array}$ & $\begin{array}{c}\text { Modelo com interação de } \\
\text { termos }\end{array}$ \\
\hline \multicolumn{4}{|c|}{$H_{1}$ - Tamanho do município } \\
\hline LP & $0,1971(0,241)$ & $0,5474(0,138)$ & $0,5573(0,207)$ \\
\hline \multicolumn{4}{|c|}{$\mathrm{H}_{2}$ - Efeito curvilíneo do tamanho } \\
\hline $\mathrm{LP}^{2}$ & ----------------- & $-0,0274(0,163)$ & $-0,0275(0,210)$ \\
\hline \multicolumn{4}{|l|}{ Capacidade do prefeito } \\
\hline $\mathbb{P}$ & $0,0979(0,537)$ & $0,0776(0,594)$ & $0,1012(0,828)$ \\
\hline EP & $0,0314(0,183)$ & $0,0296(0,186)$ & $0,0278(0,661)$ \\
\hline \multicolumn{4}{|l|}{$\mathrm{H}_{3}$ - Efeitos moderadores } \\
\hline$L P^{*} \mid \mathrm{P}$ & 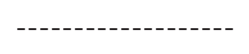 & ---------- & $-0,0025(0,950)$ \\
\hline$L P^{*} E P$ & 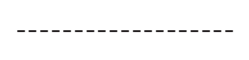 & ----------------- & $0,0002(0,981)$ \\
\hline \multicolumn{4}{|l|}{ Variáveis de controle } \\
\hline AP & $-0,1926(0,324)$ & $-0,1933(0,323)$ & $-0,1933(0,323)$ \\
\hline \multirow[t]{2}{*}{ Ex.P } & $0,0874(0,176)$ & $0,0889(0,174)$ & $0,0890(0,175)$ \\
\hline & $0,0033(0,404)$ & $0,0037(0,381)$ & $0,0037(0,383)$ \\
\hline \multicolumn{4}{|l|}{ Regressão Linear } \\
\hline$N^{0}$ Observ. & 3.504 & 3.504 & 3.504 \\
\hline$F$ & 6,04 & 5,48 & 5,74 \\
\hline Prob. $>F$ & 0,0000 & 0,0000 & 0,0000 \\
\hline$R^{2}$ & 0,0021 & 0,0025 & 0,0025 \\
\hline
\end{tabular}

Nota: ${ }^{* * *} \mathrm{p}<0,10 ;{ }^{* *} \mathrm{p}<0,05 ;{ }^{*} \mathrm{p}<0,01$.

Fonte: Dados da pesquisa. 
Observa-se que, apesar de o modelo estar ajustado ao que se propõe (Prob. $>F=0,0000$ ), o poder de explicação é muito baixo $\mathrm{R}^{2}$ 0,002, ou seja, o modelo explica $0,2 \%$ da variável dependente. Deste modo, com base na variável dependente percentual do IPTU sobre a receita fiscal total do município, devem-se rejeitar as hipóteses propostas $\left(\mathrm{H}_{1}, \mathrm{H}_{2}\right.$ e $\left.\mathrm{H}_{3}\right)$ para esta pesquisa.

Comparando com o artigo base, Avellaneda e Gomes (2015), corrobora-se com o resultado de rejeitar a $\mathrm{H}_{2}$, reforçando que o tamanho populacional não é curvilineamente associado ao desempenho municipal. Já quanto a $\mathrm{H}_{1}$ e $\mathrm{H}_{3}$, diverge-se dos resultados encontrados por Avellaneda e Gomes (2015), visto que o tamanho da população não está relacionado com o aumento do desempenho; e o aumento do tamanho da população associado com o aumento da capacidade humana do prefeito não está relacionado com o aumento no desempenho do IPTU.

A relação não significativa do tamanho do município com o aumento no desempenho aproxima-se da pesquisa de Dean e Peroff (1977), ao evidenciarem que o efeito do tamanho no desempenho municipal é neutro.

Por fim, ressalta-se que nos modelos 1 e 2 a capacidade humana do prefeito medido pela sua idade não está relacionada significativamente com o aumento do desempenho; no modelo 1 , nas três formas de análise, o alinhamento partidário é significativamente oposto (10\%) ao aumento do desempenho; em duas das formas de análise, a reeleição do prefeito está negativa e significativamente (10\%) relacionada com o aumento do desempenho; e a variável de controle não se relaciona com o aumento do desempenho.

Estes resultados divergem dos apresentados por Avellaneda e Gomes (2015), visto que não houve significância estatística nos dados. Observando a relação inversa entre o AP e, ressalta-se que o alinhamento partidário poderá facilitar na negociação entre município e governo na transferência de recursos, mas não na arrecadação de receita própria. Com base na literatura, sabe-se que quanto mais próximo da população, maior será o desempenho do município (Holzer et al., 2009), neste sentido, percebendo o governo do estado afastado da população, o alinhamento partidário entre o prefeito e o governador não reflete na arrecadação de receita própria dos municípios.

\section{CONCLUSÃO}

A pesquisa teve por objetivo analisar qual o efeito do tamanho no desempenho municipal dos municípios catarinenses. A amostra analisada foi de 293 municípios contemplando três períodos eleitorais (12 anos). Adicionalmente, verificou-se se a capacidade do prefeito afeta o desempenho municipal, quando associado ao tamanho. O desempenho municipal foi medido por duas variáveis, sendo o IPTU arrecadado per capita e o percentual do IPTU arrecadado sobre a receita fiscal total.

Com base nos resultados apresentados, nota-se que quando o desempenho é medido pelo IPTU arrecadado per capital, há influência direta do tamanho do município no desempenho. Logo, quanto maior a população do município, maior será o desempenho na arrecadação municipal. Percebe-se, também, que o tamanho influencia o desempenho municipal de forma indireta, quando associado à escolaridade do prefeito, ou seja, quanto maior o tamanho do município e quanto maior for a escolaridade do prefeito, maior será o desempenho municipal.

Esses resultados corroboram com Avellaneda e Gomes (2015) e contribuem para a discussão literária sobre o efeito do tamanho no desempenho dos municípios, visto que várias pesquisas abordam o tema (Avellaneda \& Gomes, 2015, 2017; Christenson \& Sachs, 1980; Dean \& Peroff, 1977; Fowler \& 
Walberg, 1991; Heck \& Mayor, 1993; Sharkansky, 1967; Smith \& Meier, 1994; Whetten, 1978). Já quando o desempenho foi medido pelo percentual do IPTU arrecadado da receita fiscal total, observa-se que não há influência direta e indireta do tamanho no desempenho.

Neste ponto, ressalta-se que não há consenso em relação ao efeito do tamanho no desempenho municipal, demandando mais esforços em pesquisas. Consequentemente, recomenda-se para pesquisas futuras: a utilização de outra amostra, tendendo a comparação entre outros estados brasileiros; separação da amostra em municípios menores, médios e maiores para comparação entre os dados; inclusão no modelo das variáveis de controle que medem o desempenho socioeconômico, visto que podem influenciar ou auxiliar a explicar o aumento no desempenho (na mesma linha da pesquisa de Damanpour et al. (2009); e a segregação de amostras estaduais por regiões, visando analisar se a localidade regional contribui para o efeito do tamanho no desempenho.

Esta pesquisa não está isenta de limitações, sendo a baixa força de explicação do modelo 2 uma delas. Além das recomendações para pesquisas futuras, alerta-se para cuidados estatísticos com problemas de autocorrelação entre as variáveis e heterocedasticidade. Modelos robustos e com alto grau de confiabilidade auxiliam no desenvolvimento literário, apontando caminhos a serem seguidos e confirmando (ou rejeitando) o efeito nas relações propostas (Fávero \& Belfiore, 2017; Gujarati \& Porter, 2011).

Por fim, retoma-se a pergunta do título da pesquisa: "Os pequenos perfumes fazem grandes frascos?” Esta pesquisa oferta indícios de que não, os pequenos perfumes não geram grandes frascos! De fato, constatou-se nesta pesquisa que quando o desempenho é medido pelo IPTU arrecadado per capital, há influência direta do tamanho do município no desempenho, ou seja, municípios maiores ganham vantagem em relação aos pequenos. Afinal, demonstrou-se nesta pesquisa que municípios maiores (com maior a população) tendem a ter maior desempenho na arrecadação municipal. Além disso, conclui-se que o tamanho do município também influencia o desempenho municipal de forma indireta, quando associado à escolaridade do prefeito, ou seja, municípios de tamanhos maiores e com maior escolaridade do prefeito, tendem em média a ter maior desempenho municipal. Para tanto, baliza-se no efeito direto e indireto do tamanho no desempenho encontrado no modelo 1. Mas, diante das limitações de pesquisas e da adoção de uma postura cautelosa, corrobora-se com Avellaneda e Gomes (2015) na afirmação de que no cenário brasileiro "permanece incerto" que o tamanho e o capital humano são a receita para o crescimento no desempenho municipal. 


\section{REFERÊNCIAS}

Adhikari, A., Derashid, C., \& Zhang, H. (2006). Public policy, political connections, and effective tax rates: Longitudinal evidence from Malaysia. Journal of Accounting and Public Policy, 25(5), 574-595.

Anderson, K. (2003). What motivates municipal governments? Uncovering the institutional incentives for municipal governance of forest resources in Bolivia. Journal of Environment \& Development, 12(1), 5-27.

Avellaneda, C. N. (2009). Municipal performance: does mayoral quality matter? Journal of Public Administration Research and Theory, 19(2), 285-312.

Avellaneda, C. N. (2012). Do Politics or Mayors' Demographics Matter for Municipal Revenue Expansion? Public Management Review, 14(8), 1061-1086.

Avellaneda, C. N., \& Gomes, R. C. (2015). Is small beautiful? Testing the direct and nonlinear effects of size on municipal performance. Public Administration Review, 75(1), 137-149.

Avellaneda, C. N., \& Gomes, R. C. (2017). Mayoral quality and municipal performance in Brazilian local governments. Organizações \& Sociedade, 24(83), 555-579.

Blair, H. (2000). Participation and accountability at the periphery: democratic local governance in six countries. World Development, 28(1), 21-39.

Burrell, G., \& Morgan, G. (1979). Sociological Paradigms and Organizational Analysis. London, UK: Heinemann Books.

Campbell, C. (1993). Public service and democratic accountability. In R. Chapman (Ed.), Ethics in Public Service (pp. 111-134). Edinburgh, Scotland: Edinburgh University Press.

Carreirão, Y. S. (2006). Ideologia e partidos políticos: um estudo sobre coligações em Santa Catarina. Opinião Pública, 12(1), 136-163.

Christenson, J. A., \& Sachs, C. E. (1980). The Impact of Government Size and Number of Administrative Units on the Capacity of Public Services. Administrative Science Quarterly, 25(1), 89-101.

Clingermayer, J. C., \& Wood, B. D. (1995) Disentangling patterns of state debt financing. American Political Science Review, 89(1), 108-120.

Damanpour, F., Walker, R. M., \& Avellaneda, C. N. (2009). Combinative effects of innovation types and organizational performance: A longitudinal study of service organizations. Journal of management studies, 46(4), 650-675.

Dean, G., \& Peroff, K. (1977). The Spending-Service cliché: an empirical reevaluation. American Politics Research, 5(4), 501-516.

Doig, J. W., \& Hargrove, E. C. (1990). Leadership and innovation: entrepreneurs in government. Baltimore, MD: Johns Hopkins University Press, 1990.

Drew, J., Kortt, M. A., \& Dollery, B. (2014). Economies of scale and local government expenditure: evidence from Australia. Administration \& Society, 46(6), 632-653.

Durant, R., \& Legge, J. S. (1993). Policy design, social regulation and theory building: lessons from the traffic safety policy arena. Political Research Quarterly, 46(1), 641-56.

Fávero, L. P., \& Belfiore, P. (2017). Manual de análise de dados: estatística e modelagem multivariada com Excel, SPSS e Stata. Rio de Janeiro, RJ: Elsevier.

Flynn, N. (1993). Public Sector Management. (2nd ed.). New York: Harvester Wheatsheaf.

Fowler, W. J., \& Walberg, H. J. (1991). School Size, Characteristics, and Outcomes. Educational Evaluation and Policy Analysis, 13(2), 189-202.

Gibson, C. C., \& Lehoucq, F. E. (2003). The local politics of decentralized environmental policy in Guatemala. Journal of Environment \& Development, 12(1), 28-49.

Gooding, R. Z., \& Wagner III, J. A. (1985). A metaanalytic review of the relationship between size and performance: The productivity and efficiency of organizations and their subunits. Administrative Science Quarterly, 30(4), 462-481.

Grosskopf, S., \& Yaisawarng, S. (1990). Economies of scope in the provision of local public services. National Tax Journal, 43(1), 61-74.

Gujarati, D. N., \& Porter, D. C. (2011). Econometria básica (5. ed.). São Paulo, SP: McGraw Hill.

Heck, R. H., \& Mayor, R. A. (1993). School characteristics, school academic indicators and student outcomes: Implications for policies to improve schools. Journal of Education Policy, 8(2), 143-154.

Hedeker, D., \& Gibbons, R. D. (2006). Longitudinal data analysis. Hoboken, NJ: John Wiley \& Sons. 
Holbrook, T. M., \& Van Dunk, E. (1993). Electoral competition in the American states. American Political Science Review, 87(4), 955-962.

Holzer, M., \& Fry, J., Charbonneau, E., Van Ryzin, G., Wang, T., ... Burnash, E. (2009). Literature review and analysis related to optimal municipal size and efficiency. Newark, NJ: Rutgers-Newark, School of Public Affairs and Administration.

Holzer, M., \& Yang, K. (2004). Mapping the terrain of public service quality improvement: twentyfive years of trends and practices in the United States. International Review of Administrative Sciences, 75(3), 15-31. Recuperado de https://doi. org/10.1177/0020852309341330

Lapsley, I. (1999). Research in public sector accounting: an appraisal. Accounting, Auditing \& Accountability Journal, 1(1), 21-33.

Lowi, T. (2009). Arenas of Power. Boulder, CO: Paradigm Publishers.

Lynn, L. E., Jr. (1996). Public Management as Art, Science, and Profession. Chatham, NJ: Chatham House.

Marenco, A., Strohschoen, M. T. B., \& Joner, W. (2017) Capacidade estatal, burocracia e tributação nos municípios brasileiros. Revista de Sociologia e Política, 25(64), 3-21. Recuperado de https://doi. org/10.1590/1678-987317256401.

Meier, K. J., \& O'Toole, L. J. (2002). Public management and organizational performance: the effect of managerial quality. Journal of Policy Analysis and Management, 21(4), 629-643.

Pandey, S. K., \& Moynihan, D. P. (2006). Bureaucratic red tape and organizational performance: testing the moderating role of culture and political support. In: Boyne, G. A. et al. (Ed.). Public Service Performance. Cambridge, UK: Cambridge University Press.

Parker, L. D., \& Gould, G. (1999). Changing public sector accountability: critiquing new directions. Accounting Forum, 23(2), 109-135.

Peters, B. G., \& Savoie, D. J. (1996). Managing incoherence: the coordination and empowerment conundrum. Public Administration Review, 56(3), 281-290.

Poi, B., \& Wiggins, V. (2001). Testing for panel-level Heteroskedasticity and autocorrelation. StataCorp LP.
Roca-Puig, V., Beltrán-Martín, I., \& Segarra Cipres, M. (2011). Combined effect of human capital, temporary employment and organizational size on firm performance. Personnel Review, 41(1), 4-22.

Romzek, B. S. (2000). Dynamics of public sector accountability in an era of reform. International Review of Administrative Sciences, 66(1), 21-44.

Sampieri, R. H., Collado, C. F., \& Lucio, P. B. (2013). Metodología de la investigación. México, DF: McGraw-Hill Interamericana.

Schumacher, E. F. (1973). Small is beautiful: a study of economics as if people mattered. New York, NY: Harper Perennial.

Sharkansky, I. (1967). Government expenditures and public services in the American states. American Political Science Review, 61(4), 1066-77.

Smith, K. B., \& Meier, K. J. (1994). Politics, bureaucrats, and schools. Public Administration Review, 54(6), 551-58.

Stein, R. M. (1990). The budgetary effects of municipal service contracting: A principal-agent explanation. American Journal of Political Science, 34(2), 471-502.

Steiner, R. (2003). The causes, spread and effects of intermunicipal cooperation and municipal mergers in Switzerland. Public Management Review, 5(4), 551-571.

Swank, D. (2002). Global capital, political institutions, and policy change in developed welfare states. Cambridge, UK: Cambridge University Press.

Van Der Hoek, M. P. (2005). From cash to accrual budgeting and accounting in the public sector: The Dutch experience. Public Budgeting \& Finance, 25(1), 32-45.

Walker, R., Damanpour, F., \& Avellaneda, C. N. (2009). Combinative effects of innovation types on organizational performance: a longitudinal study of public services. Journal of Management Studies, 46(4), 650-675.

Whetten, D. A. (1978). Coping with incompatible expectations: an integrated view of role conflict. Administrative Science Quarterly, 23(2), 254-71. 


\section{Filipy Furtado Sell}

https://orcid.org/0000-0003-4335-4055

Doutor em Contabilidade pela Universidade Federal de Santa Catarina (UFSC); Professor da Faculdade de Ciências Contábeis da Universidade Federal do Pará (UFPA). E-mail: filipysell@ufpa.br

\section{Leonardo Flach}

https://orcid.org/0000-0002-4316-0704

Pós-Doutor em Contabilidade e Finanças pelo Massachusetts Institute of Technology (MIT/EUA); Professor do Programa de Pós-Graduação em Contabilidade da Universidade Federal de Santa Catarina (UFSC).

E-mail: leonardo.flach@ufsc.br

\section{Luísa Karam de Mattos}

https://orcid.org/0000-0003-1990-3034

Doutoranda em Administração pela Universidade Federal de Santa Catarina (UFSC); Mestre em Administração pela Universidade Federal de Santa Catarina (UFSC). E-mail: luisakmattos@gmail.com 